\title{
Ambiente de desarrollo virtual para el aprendizaje de la programación: un estudio de caso en la Lic. de Sistemas de la Universidad Nacional de Río Negro, Patagonia Argentina
}

\author{
Edith Lovos, Universidad Nacional de Río Negro, Argentina
}

\begin{abstract}
Resumen: En este artículo se presentan los resultados obtenidos y conclusiones alcanzadas a través de la implementación de una estrategia de enseñanza y aprendizaje, diseñada para el desarrollo de las actividades de laboratorio de un primer curso de programación. La misma se basa en la aplicación del trabajo colaborativo usando un entorno de desarrollo, que combina algunas funcionalidades provistas por el entorno Moodle y un módulo que se integra al mismo, llamado Virtual Programming Lab (VPL). Los futuros profesionales de Sistemas, por su especificidad laboral, incluirán y utilizarán las denominadas tecnologías de la información y la comunicación (TIC). Así mismo, en el ejercicio profesional, la actividad de desarrollo del software, requiere del trabajo en equipo y en colaboración. Por todo esto, resulta necesario contemplar estos requerimientos en la formación de los alumnos de sistemas, desde los inicios de su formación.
\end{abstract}

Palabras clave: programación, colaboraciòn, TIC

\begin{abstract}
This article presents the results obtained and conclusions reached through the implementation of a strategy of teaching and learning, designed for the development of the laboratory activities for a first programming course. The same is based on the application of the collaborative work using a development environment, which combines some functionality provided by the learning management system, Moodle and a module that integrates the same, called Virtual Programming Lab (VPL). Future professionals in computer science by its specificity labor, will include and use the so-called information and communication technologies (ICT). Likewise, in the professional practice, the activity of software development, requires teamwork and collaboration. By all this, it is necessary to consider these requirements in the training of students of computer science, from the beginning of their training.
\end{abstract}

Keywords: Programming, Collaboration, ICT

\section{Introducción}

$\mathrm{E}$ ste artículo presenta algunos de los resultados alcanzados en la primera implementación de una estrategia de enseñanza y aprendizaje, basada en el trabajo colaborativo y mediada por recursos informáticos para un curso introductorio de programación de computadoras de nivel universitario. La experiencia ha sido diseñada para trabajar específicamente las actividades de laboratorio del curso; con la intención de potenciar el aprendizaje de la programación y promover en los alumnos habilidades requeridas en el ámbito laboral actual, como el trabajo en equipo, la capacidad de comunicación y el "aprender a aprender".

La investigación buscó abordar el proceso de enseñanza y aprendizaje de las ciencias de la computación atendiendo a tres factores:

- la complejidad asociada al proceso de resolución de problemas a través de la algoritmia.

- el escenario educativo actual, donde el aprendizaje se entiende como un proceso social, que se construye a través de la interacción de diferentes actores (docentes - alumnos, alumnos - alumnos) y donde el contexto y el significado que cada uno le asigna a lo que aprende adquiere suma importancia. (Zañartu, 2003; Maldonado, 2007).

- y la formación del futuro profesional de sistemas, como parte de una sociedad, que se encuentra atravesada por las denominadas Tecnologías de la Información y la Comunicación (TIC).

Revista Internacional de Ciencia, Matemáticas y Tecnología

Volumen 2, Número 1, 2015, <http://sobrelaeducacion.com>, ISSN 2386-8781

CGlobal Knowledge Academics. Edith Lovos.

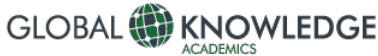

soporte@gkacademics.com 
En el ámbito laboral y profesional de la industria del software, el proceso de producción del mismo se presenta como una actividad compleja que resulta impensable desarrollarla en soledad y que por el contrario, requiere de la colaboración de grandes equipos de personas. Así una de las habilidades requeridas por las empresas dedicadas a esta industria, es que el desarrollador de software tenga capacidad de trabajo en equipo y la habilidad de "aprender a aprender". Por otra parte, la actividad profesional se encuentra atravesada no solo por las TIC sino por un nuevo paradigma de trabajo denominado Desarrollo Global del Software (DGS), donde una de sus características es que los miembros de un grupo de trabajo puedan estar distribuidos geográficamente. (Monazor et al., 2011). De esta forma, a partir de esta investigación se buscó demostrar que resulta necesario desarrollar en los futuros profesionales de sistemas, habilidades y destrezas que les permitan incorporarse a ese nuevo espacio laboral. Y en este sentido, el concepto de trabajo colaborativo se presenta como una posibilidad para el desarrollo de las actividades de formación académica y como una forma de llevar adelante la actividad profesional.

\section{Marco teórico}

En los trayectos de formación de las Ciencias de la Computación, la enseñanza de la programación constituye un pilar fundamental y uno de los primeros cursos que necesariamente, deben tomar los alumnos ingresantes a estas carreras universitarias (Matthíasdóttir, 2006). Son varios los estudios que permiten afirmar que la enseñanza y aprendizaje de la disciplina en estos cursos, resulta una actividad intelectual compleja y dificultosa no solo para los estudiantes, sino también para quienes tienen a cargo la enseñanza, sobretodo cuando su impacto es muy importante en la mayoría de las asignaturas sucesivas y en el campo profesional del futuro egresado. (Costelloe, 2001; Lahtinen et al., 2005; Matthíasdóttir, 2006; DiGiusti et al., 2003; Madoz et al., 2005).

Los cursos de programación en la formación de un profesional de sistemas, buscan que los alumnos desarrollen a lo largo de su formación habilidades para la resolución de problemas y estrategias efectivas para el diseño e implementación de las soluciones a los mismos. (Ala-Mutka, 2006).

\section{Trabajo colaborativo \& aprendizaje de la programación}

El trabajo colaborativo está asociado al conjunto de métodos de instrucción y entrenamiento, que buscan promover el desarrollo de habilidades como aprendizaje, desarrollo personal y social; y la responsabilidad no solo a nivel individual sino colectiva. (Lucero, 2003). Un concepto clave, en esta forma de trabajo, es el denominado por Vygostky, como "zona de desarrollo próximo", entendiéndola como aquella zona situada entre lo que un estudiante puede lograr solo y lo que puede lograr si trabaja con la guía de un instructor o en colaboración con otros pares más avanzados. (Vygostky, 1978). Por otra parte, la colaboración en el ámbito académico, nace y responde a un nuevo contexto (social y cultural), donde se define el "cómo aprendemos" (socialmente) y "dónde aprendemos" (en red). (Zañartu, 2003).

Con la intención de mejorar la enseñanza y el aprendizaje de la programación, en el nivel universitario, se han diseñado diversas estrategias, que van desde aquellas manejadas por tecnología como la visualización del software, el uso de robots o las herramientas de diseño hasta aquellas que involucran paradigmas de enseñanza como el aprendizaje basado en problemas, aprendizaje cognitivo o aprendizaje basado en patrones o casos. (Costelloe, 2001). Dónde el rasgo característico de las estrategias consiste en promover la construcción del conocimiento por parte del proprio individuo, en interacción con su entorno. De esta forma, el alumno necesita construir, testear y refinar representaciones cognitivas que le permitan darle sentido al contexto. De allí, la influencia del constructivismo en el aprendizaje de las ciencias de la información y la computación.(Jiménez Builes et al., 2008).

Teague (2008), sostiene que la aplicación de estrategias de enseñanza colaborativa, en materias vinculadas a la programación, promueven en los alumnos, un compromiso más fuerte y activo, los alienta a pensar en voz alta y a verbalizar el proceso de resolución de problemas, satisfaciendo de esta forma, la necesidad de interacción de los alumnos actuales. Por su parte, el uso de los ambientes 
colaborativos (CSCL) en el desarrollo de las actividades de enseñanza y aprendizaje de la programación, le ofrecen a los alumnos a través de un soporte computacional, la posibilidad de beneficiarse del conocimiento y habilidades del resto de integrantes del grupo, mejorando así sus propias destrezas.(Jurado et al., 2012). De esta forma, el aprendizaje a través de estos ambientes, busca promover espacios para el desarrollo de habilidades a nivel individual y grupal que tienen lugar a partir de la discusión que se genera entre los alumnos de un grupo de trabajo al momento de explorar nuevos conceptos. (Lucero, 2003).

A nivel profesional, las herramientas comerciales que se utilizan para el desarrollo del software, presentan una amplia cantidad de opciones y de información que los alumnos que recién se inician en la práctica de la programación no pueden comprender tan fácilmente porque aún no tienen los conceptos necesarios para manipularlas (Pérez et al., 2006). En este sentido, resulta interesante, que los entornos de desarrollo que se utilicen para la enseñanza y el aprendizaje de la programación, provean a los alumnos de un ambiente que les facilite las tareas relacionadas con el desarrollo del software.

En un artículo anterior (Lovos, 2010), se ha realizado una revisión de tres herramientas que pueden usarse como soporte para el desarrollo colaborativo de la programación en el ámbito educativo: EclipseGavab, COLLEGE y Virtual Programming Lab.

\section{Virtual Programming Lab}

Virtual Programming Lab (VPL) es un aplicativo de código abierto, creado por el Departamento de Informática y Sistemas, de la Universidad de Las Palmas de Gran Canaria.

VPL, permite la gestión de las prácticas de programación a través del entorno Moodle, posibilitando de esta forma la inclusión del ambiente de desarrollo al aula virtual de las materias donde se utiliza. Su arquitectura está compuesta de un módulo Moodle, un applet editor de código fuente y un proceso especial Linux, comúnmente llamado demonio, que permite la ejecución remota de programas de forma segura.

La intención de VPL es facilitar el seguimiento y la orientación personalizada y continua del proceso de aprendizaje del alumno, contribuyendo de este modo, a tratar las dificultades a las que se enfrenta éste en la realización de las actividades de programación. VPL busca proveer a los alumnos (en particular a los que recién se inician con las actividades de programación), de un entorno de desarrollo, que les resulte simple y fácil de utilizar. Entre sus características más destacadas se pueden mencionar: la posibilidad de editar el código fuente y ejecutar las prácticas de forma interactiva desde el navegador, ejecutar pruebas que revisen las prácticas y analizar la similitud entre prácticas para el control del plagio (Rodriguez et al., 2010). Por otra parte, permite realizar:

- Control de entregas: permite limitar el periodo de entrega y el acceso a la tarea. Es posible limitar la cantidad de archivos que se adjuntan en las entregas y el tamaño de los mismos. Es posible restringir las operaciones de visualización, edición y subida de archivos, a las redes que se especifiquen o protegerlos a través del uso de contraseñas.

- Gestión de archivos: la edición de los archivos se realiza desde el propio navegador, para lo cual se emplea un editor de código applet java componente del sistema. Es posible establecer contenidos iniciales en los archivos de entregables. De esta forma, cuando el alumno accede al editor éste estará precargado con contenido preestablecido.

- Ejecución y Evaluación de la práctica: es posible definir scripts y programas para evaluar las actividades. Se puede ejecutar el programa con entrada y salida en una consola en el navegador. También permite imponer límites a los recursos empleados en la ejecución: tiempo, memoria, tamaño de los archivos y cantidad de procesos.

\section{Estrategia de enseñanza y aprendizaje}

La estrategia de enseñanza y aprendizaje que se discute en este artículo, se denominó Actividades Prácticas Entregables (APE) . Las mismas consistieron en la resolución de problemas computacionales de mediana complejidad, a través del trabajo en equipos colaborativos tutorizados por un do- 
cente. El proceso de resolución de las APE, estuvó orientado a promover la participación de los alumnos, el desarrollo de competencias tales como el razonamiento crítico, el trabajo en equipo y la comunicación. Así como también que los alumnos puedan apropiarse de las TIC, como un recurso para el aprendizaje.

El proceso de resolución de las APE se divide en 5 etapas: Debate Inicial, Análisis y Diseño, Implementación, Presentación y Defensa, y Evaluación. Y como soporte tecnológico al mismo, se propuso el uso de un ambiente de trabajo, que combina algunas funcionalidades provistas por el entorno Moodle (foros y wiki principalmente) y VPL. Dónde cada grupo disponía de una instancia de ese ambiente de trabajo, y cada participante solo podía manipular la correspondiente a su grupo. La tabla 1, muestra para cada etapa del proceso de resolución de las APE, los recursos TIC propuestos como soporte.

Tabla 1: APE - Etapas de desarrollo

\begin{tabular}{|l|l|}
\hline Etapa & Recursos TIC \\
\hline Debate Inicial & $\begin{array}{l}\text { Presentación Consigna sobre Aula Virtual. (archivo pdf). } \\
\text { Foro }\end{array}$ \\
\hline Análisis \& Diseño & Wiki, Foro \\
\hline Implementación & Wiki, Foro, VPL \\
\hline Presentación \& Defensa & $\begin{array}{l}\text { Wiki, Foro } \\
\text { Presentación de la producción grupal (.ppt o similar) }\end{array}$ \\
\hline Evaluación & Encuesta embebida en el aula virtual \\
\hline
\end{tabular}

Fuente: Información adaptada de Lovos, 2014.

El ambiente de trabajo propuesto, permitió integrar las actividades de análisis y diseño de la solución algorítmica con las actividades de implementación, es decir la codificación, compilación y ejecución del programa computacional que resuelve el problema. Así mismo, el ambiente permitió igualar las condiciones de implementación del programa computacional, ya que todos los equipos podían hacer uso del mismo editor y compilador, sin necesidad de descargar ningún aplicativo extra; solo necesitaban habilitar en el navegador desde el cual accedían al aula virtual del curso, el plugin de Java que permite que se ejecute el software VPL.

Un punto a resaltar de la estrategia propuesta, es el proceso de evaluación. El mismo, consta de tres partes: la evaluación del programa computacional (a través del software VPL), una evaluación en forma presencial a modo de exposición y defensa de la solución propuesta y una tercera evaluación a modo de encuesta a través del aula virtual, que permitió que los alumnos evalúen el proceso de desarrollo de la APE, valorando su propio desempeño, el de su grupo de trabajo y el del tutor asignado. De esta forma, la fase de evaluación no solo buscó evaluar el resultado final de la APE (programa computacional) sino también los mecanismos que permitieron el desarrollo de la propuesta.

\section{Implementación}

En esta sección se describe el contexto donde se llevo a cabo la primera implementación de la propuesta de enseñanza y aprendizaje, así como también la metodología de investigación utilizada y un análisis de algunos de los resultados obtenidos.

\section{Contexto}

La primera experiencia de uso de la estrategia APE, se realizó en un curso de primer año de la Lic. en Sistemas de la UNRN, correspondiente a la materia Programación de Computadoras I (PCI). El mismo, tuvo lugar durante el primer cuatrimestre del año lectivo 2013. La UNRN es una Universidad Nacional de la Patagonia Argentina, creada en el año 2007; y desde el inicio de sus actividades académicas en el año 2009, persigue en su proyecto institucional avanzar hacia la incorporación de las enseñanzas mediadas por TIC en sus propuestas formativas. En este sentido, en la Lic. de Siste- 
mas, desde sus comienzos se ha dispuesto de un espacio virtual de soporte a la clases presenciales, sobre el entorno Moodle.

A través de la materia PCI, se busca que los alumnos puedan analizar problemas resolubles con computadora, poniendo énfasis en la modelización, la abstracción de funciones y en la descomposición funcional de los mismos, a partir de un paradigma procedural/ imperativo. Así como también, introducir algunas nociones de estructuras de datos, tipos de datos y abstracción de datos.

El curso es de carácter presencial, con un total de 96 horas; dividido en clases teóricas y prácticas, que se dictan en forma semanal. En las primeras se desarrollan los conceptos teóricos previstos en el plan de estudio (resolución de problemas, estructuras de control, modularización, estructuras de datos) haciendo uso de ejemplos prácticos que permitan la aplicación de los conceptos analizados. Respecto a las clases prácticas, las mismas tienen como objetivo la aplicación de los conceptos trabajados en las clases de teoría, en la resolución de problemas computacionales, a través del diseño algoritmos y la implementación en un lenguaje de programación de alto nivel tipo Pascal. El énfasis de la asignatura está puesto en la parte práctica, ya que para desarrollar la habilidad de resolver problemas usando algoritmos resulta fundamental el entrenamiento. El programa consta de seis unidades didácticas, cada una con su correspondiente trabajo práctico y tres prácticas de laboratorio usando Actividades Prácticas Entregables (APE).

La tabla 2, muestra para cada APE las unidades temáticas que se abordaron y las consignas planteadas.

Tabla 2: Consignas y grupos por APE

\begin{tabular}{|l|l|l|}
\hline APE & Unidades Temáticas & Consignas \\
\hline APE1 & $\begin{array}{l}\text { Introducción a Pascal. Estructuras } \\
\text { de Control, Datos y Tipos de Datos }\end{array}$ & $\begin{array}{l}\text { Juego “La Tapadita” } \\
\text { Simulación "Teclado telefónico" }\end{array}$ \\
\hline APE2 & $\begin{array}{l}\text { Tipos de Datos indexados.Vectores } \\
\text { y Matrices }\end{array}$ & $\begin{array}{l}\text { Juego Ta-Te-TI } \\
\text { Simulación Cuadrado Mágico }\end{array}$ \\
\hline APE3 & $\begin{array}{l}\text { Punteros. Estructuras de Datos } \\
\text { Compuestas. Listas Enlazadas }\end{array}$ & $\begin{array}{l}\text { Simulación Pilas } \\
\text { Simulación Colas }\end{array}$ \\
\hline
\end{tabular}

Fuente: Información adaptada de Lovos, 2014.

El curso PCI dispone de un aula virtual sobre el entorno Moodle, a la cual pueden acceder solo los alumnos y docentes matriculados al mismo. El aula virtual permite que los docentes publiquen allí los materiales de las clases teóricas y prácticas, y que los alumnos encuentren otro espacio para la comunicación con sus pares y docentes, más allá del espacio presencial.

Los alumnos que se inscriben al curso, son en su mayoría ingresantes a la universidad, cuyas edades varían entre los 17 y 21 años. Y dónde para la mayoría de ellos, este curso, es su primer contacto con la actividad de programación. En relación a su vinculación con la tecnología, es común verlos, utilizando teléfonos celulares inteligentes que les permiten acceder a internet, escuchar música o mirar videos. Asimismo, varios de ellos llegan al curso con computadoras portátiles. Todo esto, hace suponer que tienen cierto manejo de las TIC (navegadores, redes sociales como Facebook entre otros).

Respecto a la organización de los grupos de trabajo, para el desarrollo de las APE, en función de la complejidad que presentan las mismas y teniendo en cuenta que desde los inicios de la carrera, en el año 2009, los alumnos inscriptos en el curso no superan los 50, en promedio, se propone que los equipos de trabajo no superen los 4 alumnos. En cuanto a su conformación, en esta primera experiencia se propuso, para la primer APE, que fueran los propios alumnos quienes organizasen los equipos, luego en las APE siguientes, los equipos fueron re-armados por los docentes de la materia de acuerdo al seguimiento realizado y en función de los requerimientos de los propios alumnos.

En relación a los docentes que participaron como tutores de las APE, los mismos fueron el docente a cargo de las clases teóricas y el docente a cargo de las clases prácticas de la materia. 


\section{Metodología}

La metodología de investigación utilizada tiene un enfoque cualitativo, que integra aspectos de tipo cuantitativo. Donde, el objetivo principal consistió en interpretar y entender como la implementación de una estrategia de enseñanza y aprendizaje basada en el trabajo colaborativo y mediada por recursos tecnológicos, (APE), puede potenciar el aprendizaje en los alumnos de la asignatura Programación de Computadoras I (PCI), de la Lic. en Sistemas de la UNRN, de la Sede Atlántica. En este sentido, se optó por un estudio de caso, con la idea de explorar, describir, explicar, evaluar y abrir un camino para posibles transformaciones en la enseñanza y aprendizaje de la disciplina.

Al curso PCI cohorte 2013, se inscribieron 64 alumnos, de los cuales el 18,75\% son mujeres y el resto son varones. Del total de inscriptos, los ingresantes corresponden al $88 \%$ y el $12 \%$ son alumnos recursantes de la cohorte 2012. La experiencia, se inició, luego de transcurridas las primeras 6 clases del curso, procurando que los alumnos pudieran entre otras cosas, familiarizarse con el aula virtual de la materia y vincularse con sus compañeros y docentes.

La recolección de los datos se realizo a través de: encuestas a los alumnos, entrevistas a docentes y alumnos, y observaciones de clase tanto en forma presencial como a través de los recursos TIC propuestos por las APE. En relación a las encuestas que respondieron los alumnos, las mismas se basaron en un modelo propuesto por el Instituto Tecnológico y de Estudios Superiores de Monterrey $^{1}$, con la intención de conocer:

- las percepciones de los alumnos en relación a sus propios procesos de aprendizaje.

- las valoraciones de los alumnos en relación al trabajo colaborativo.

- la evaluación de los alumnos respecto a la enseñanza.

Las encuestas se aplicaron en cada una de las APE (en la etapa de evaluación de la misma) y se realizaron al total de los alumnos participantes de la experiencia. Las mismas fueron anónimas, a nivel individual y estuvieron accesibles desde el aula virtual de la materia sobre la que se realizó la experiencia. Esto último, posibilitó la recolección automática de los datos. Respecto a su contenido, se utilizo un cuestionario estructurado en tres ejes: preguntas de autoevaluación, preguntas para evaluar el trabajo en equipo y preguntas respecto al proceso de enseñanza durante el desarrollo de las APE. En cuanto al tipo de preguntas, se incluyeron:

- cerradas: limitadas a un conjunto de opciones, con la intención de facilitar la categorización y codificación de las respuestas.

- abiertas: a modo de texto, donde el encuestado podía desarrollar la respuesta.

En relación al tamaño de las encuestas, para la APE 1 y 2 , tuvieron un total de 22 preguntas y para la última APE, 26 preguntas. Las preguntas que se agregaron a la última encuesta, buscaban conocer si el desarrollo de las APE, les había permitido a nivel individual, prepararse mejor para el examen, si les gustaría que la forma de trabajo desarrollada a través de las APE se aplicará a otras asignaturas de la carrera y por último saber que cambios consideraban que podrían hacerse en la materia. El diseño de las encuestas está disponible en http://sedici.unlp.edu.ar/handle/10915/38038 .

En cuanto a las entrevistas a los alumnos, las mismas se realizaron al finalizar la experiencia, con la intención de contrastar información proveniente de las encuestas, de las observaciones de clase y de los recursos TIC propuestos como apoyo virtual a la estrategia. Así mismo, se realizaron tanto en forma grupal como individual.

\section{Análisis de los resultados}

A continuación, se presentan algunos de los resultados alcanzados a partir del material recolectado.

En la figura 1, se pueden visualizar las percepciones de los alumnos respecto a si el desarrollo de las APE les permitió una mejor comprensión a nivel individual, de los conceptos involucrados en las mismas. Aquí, más del $50 \%$ de los alumnos, consideran que la estrategia propuesta, contribuyo en

\footnotetext{
${ }^{1}$ Instituto Tecnológico y de Estudios Superiores de Monterrey. "El aprendizaje basado en problemas como técnica didáctica". http://www.ub.edu/mercanti/abp.pdf. 2000
} 
forma normal en las dos primeras APE y en la última se observa que la contribución superó el 70\%. La tercer APE, resultó una experiencia de investigación para los grupos, ya que la resolución del problema planteado requirió de conceptos no analizados en clase (como es el caso de las estructuras de datos Pilas y Colas). En este sentido, los grupos realizaron un aprendizaje de las temáticas planteadas a través del descubrimiento y construcción del mismo, ya que fueron los propios alumnos quienes tuvieron que buscar información, seleccionarla, organizarla e intentar resolver el problema planteado en la consigna de la APE. Contando en todo momento con la orientación del tutor, pero donde la resolución del problema estuvo sujeta a las elecciones realizadas por el grupo. (Restrepo, 2005)

Figura 1: APE's Temas. Nivel de Comprensión

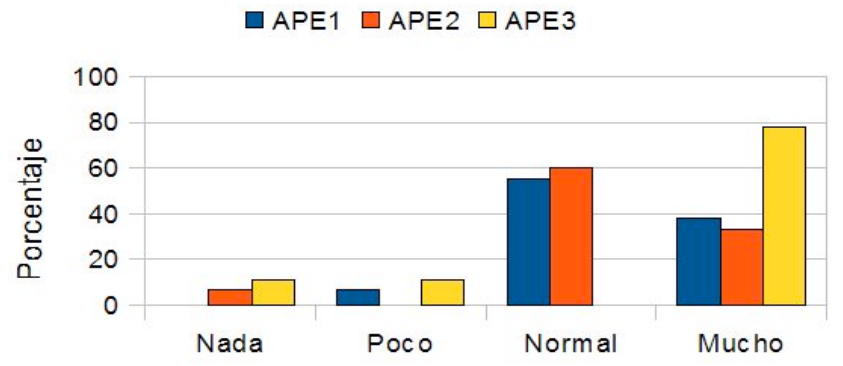

Fuente: Lovos, 2014.

En relación al trabajo en equipo, consultados en las tres encuestas si volverían a trabajar con el mismo equipo se obtuvieron los resultados que se muestran en el gráfico 2, dónde se observa que en general la tendencia fue positiva, salvo para la APE 2. En este sentido, consultados los alumnos sobre que consideraban había sido lo mejor y lo peor de trabajar con su equipo de trabajo, señalaron en general, la falta de conocimientos y comprensión de la situación problemática planteada. Así un alumno de un grupo de la APE 2, señalaba: "La falta de conocimiento y comprensión de todo el grupo, (me incluyo) hizo que se tardara en resolver el APE2. Personalmente tuve q estudiar mucho, leer y mirar tutoriales en Internet para poder comprender realmente la manera de trabajar con los nuevos conocimientos".

Figura 2: APE's Consolidación grupal

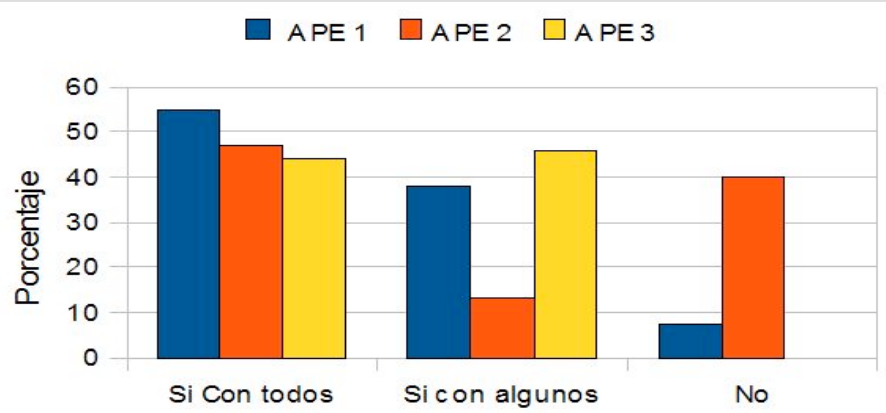

Fuente: Lovos, 2014.

En cuanto a la etapa de presentación y defensa de las APE, resulta importante destacar cómo evolucionaron a lo largo de la experiencia, las presentaciones digitales que realizaron los grupos y como los mismos interactuaron no solo con los docentes sino con los demás grupos, en la defensa oral de las actividades. Para las ponencias de las APE 1 y 2, las presentaciones fueron del estilo PowerPoint, dónde todas se caracterizaron por ubicar el foco en la implementación (código fuente en Pascal) de la solución al problema, hasta pasar a la presentación de la APE3, donde el foco se puso más sobre el modelo de la solución que sobre el código y donde algunos grupos se animaron a 
innovar en el uso de aplicativos más complejos como es caso de Prezi ${ }^{2}$, que permiten la colaboración para el desarrollo de presentaciones.

En relación al desarrollo de las ponencias, los alumnos manifestaron a través de las entrevistas, que cuando llegaban a la etapa de presentación, se incrementaba la participación en el grupo, y entienden que esto era así porque habían logrado resolver el problema que planteaba la actividad y esto los ubicaba en una situación más homogénea con respecto a los conocimientos sobre la temática planteada a través de la APE.

A este análisis, se suma la información recolectada a través del aula virtual, respecto al uso de los recursos TIC propuestos (foro, wiki, VPL). Así, en la tabla 3 se indica para cada APE, el porcentaje de utilización de cada una de esas herramientas. Dónde, es posible observar que la herramienta menos utilizada, es la wiki. En este sentido, los alumnos expresaron a través de comentarios en forma anónima y en las entrevistas de cierre de la experiencia, que no podían encontrarle utilidad a la misma para el desarrollo de las actividades de análisis y diseño de la solución algorítmica. Así mismo, remarcaron que su utilización no les resulto intuitiva y en algunos casos solo pudieron utilizar la funcionalidad que permitía subir y compartir archivos.

Tabla 3: APE's - Uso de los recursos TIC

\begin{tabular}{|l|l|l|l|}
\hline Recursos TIC & APE1 & APE2 & APE3 \\
\hline Wiki & $50,00 \%$ & $28,57 \%$ & 0 \\
\hline Foro & $100,00 \%$ & $57,14 \%$ & $54,55 \%$ \\
\hline VPL & $100,00 \%$ & $100,00 \%$ & $100,00 \%$ \\
\hline
\end{tabular}

Fuente: Información adaptada de Lovos, 2014.

En relación a las herramientas foros y wiki de Moodle, se pudo observar que al principio de la experiencia, fueron consideradas por los alumnos como un instrumento de evaluación. En decires de un alumno "La herramienta del foro o la wiki no es algo muy útil para nosotros pero entiendo que para los profesores es importante para poder evaluar a los alumnos porque personalmente no pueden ver lo que hace cada uno". Sin embargo, al finalizar la experiencia, hubieron quienes pudieron apropiarse del foro como un recurso útil no solo para el desarrollo de la APE sino para el desarrollo del resto de las actividades del curso.

\section{Conclusiones y trabajos futuros}

En relación a la forma de trabajo colaborativa, para la mayoría de los alumnos ingresantes, la misma resultó una experiencia novedosa, así como también el uso de los recursos TIC propuestos. En relación a esto último, es posible concluir que resultó difícil para los grupos adoptar las herramientas foros y wiki del entorno Moodle, para el desarrollo de las actividades didácticas específicas del área de programación, no así el uso del laboratorio virtual VPL. Esta herramienta la apropiaron como un recurso que les permitió compartir la actividad de implementación (programación) con sus compañeros y como un mecanismo de comunicación, que les posibilitó sacarse dudas y realizar consultas más allá del espacio presencial de clases.

La forma de trabajo propuesta a través de las APE, fomentó la participación de los alumnos, y la capacidad de comunicación. Esto se puso en evidencia en la etapa de presentación y defensa de las soluciones encontradas, donde se observó como mejoro en los grupos, la habilidad de exponer con claridad las ideas, la predisposición al intercambio de opiniones y la sensación de seguridad que les permitió superar miedos e inhibiciones que manifestaron al inicio de la experiencia. Esta etapa, posibilitó que los docentes pudieran recolectar información para hacer ajustes en los procesos de enseñanza y aprendizaje, teniendo en cuenta, que uno de los puntos claves para el éxito de la propuesta, está puesto en la retro-información que los grupos de trabajo obtienen en cada una de las fases del

\footnotetext{
${ }^{2}$ http://prezi.com/
} 
desarrollo y aquí se observa la importancia del docente como tutor y de los alumnos como pares avanzados dentro de los grupos.

Volviendo sobre los recursos TIC propuestos como apoyo a la estrategia de enseñanza y aprendizaje, una de las dificultades asociada al uso de la wiki de Moodle, esta relacionada con la gestión de los usuarios. Para cada una de las APE, se creaba una wiki con la intención de desarrollar sobre la misma el análisis y diseño del problema, y sucedía que las páginas de las wikis quedaban accesibles a todos los grupos de trabajo, lo cual generó confusión y miedo al plagio entre los alumnos. Estas percepciones por parte de los alumnos como de los docentes que participaron de esta experiencia, coinciden con lo analizado por otros autores. (Córdoba et al., 2009).

En función de los resultados alcanzados con esta primera experiencia, se propone:

- Ajustar el diseño de la propuesta, teniendo en cuenta las observaciones que hicieron los alumnos y los docentes de la cátedra. En particular sobre el tipo de problemas a resolver y sobre los recursos TIC propios de Moodle, como foros y wiki.

- Analizar como las APE, podrían utilizarse como una estrategia para sostener a los alumnos a lo largo de la cursada, teniendo en cuenta el desgranamiento que se observa en la materia, desde los inicios de la carrera.

- Articular esta forma de trabajo con las materias del área de Algoritmos y Lenguajes, y de Ingeniería de Software, dando a los alumnos una visión más acabada del desarrollo colaborativo de un producto de software.

Actualmente se está llevando a cabo una segunda experiencia, en la materia Programación de Computadoras II, que es correlativa de PCI y que se dicta en el segundo cuatrimestre del primer año de la carrera. Así mismo, se continúa la investigación en los temas aquí planteados a través de un proyecto de investigación acreditado por la Universidad Nacional de Río Negro, denominado "Tecnologías de la Información y la Comunicación en la Enseñanza y Aprendizaje en el Nivel Superior. Habilidades de Autoregulación de los Aprendizajes y Trabajo Colaborativo".

\section{Agradecimientos}

Los resultados y conclusiones que se presentan en este artículo provienen de una investigación llevada a cabo en la Universidad Nacional de Río Negro, a través de una beca de formación de posgrado Res. 292/2011, en el periodo 2011 - 2013, bajo la dirección del Mgter. Rodolfo Bertone (UNLP) y de la Dra. Inés Fernández Mouján (UNRN). 


\section{REFERENCIAS}

Beltran Silva, E.E., Morales Hernández, I. (2011). Autonomía y Trabajo Colaborativo. XII Congreso Internacional de Teoría de la Educación. Universidad de Barcelona. Disponible: www.cite2011.com/Comunicaciones/Escuela/152.pdf

Collazos, C. A., Guerrero, L. y Vergara, A. (2001). Aprendizaje Colaborativo: un cambio en el rol del profesor. http://www.dcc.uchile.cl/ luguerre/papers/CESC-01.pdf

Córdoba Torrecilla, J. y Cuesta Morales P. (2009). Adaptando un sistema de Wikis para su uso educativo. XV Jornadas de Enseñanza Universitaria de la Informática. JENUI 2009. Barcelona, 8-10 de julio de 2009.

Costelloe, E. (2001). Teaching Programming. The State of the Art. Department of Computing, Institute of Technology Tallaght, Dublin 24. CRITE Technical Report, 2004a. https://www.scss.tcd.ie/disciplines/information_systems/crite/crite_web/publications/sourc es/programmingv1.pdf

De Giusti, A. E., Madoz, M. C., Gorga, G., Feierherd, G. E., \& Depetris, B. O. (2003). Enfoques y herramientas en la enseñanza de un primer curso de computación (CS1). En IX Congreso Argentino de Ciencias de la Computación.

González de Rivera Fuentes, M., Paredes Velasco, M. (2008). Aprendizaje con Programación Colaborativa. Número 2008 - 02. Serie de Informes Técnicos DLSI1-URJC. ISSN 1988-8074. Departamento de Lenguajes y Sistemas Informáticos I Universidad Rey Juan Carlos.

Jiménez Builes J.A., Pavony Meneses M., Alvarez Serna, A. F. (2008). Entorno de integración de PBL y CSCL para la enseñanza de algoritmos y programación en ingeniería. Avances en Sistemas e Informática, 5(3), pp. 189-194. Disponible en: http://www.revista.unal.edu.co/ index.php/avances/article/view/10112 Junio 2012

Jurado, F., Molina A. I., Redondo, M. A. y Ortega. D. M. (2012). Cole-Programming: Incorporando Soporte al. Aprendizaje Colaborativo en Eclipse. IEEE-RITA, 7(3), pp. 121-130.

Lahtinen E, Ala-Mutka K. et al. (2005). A Study of the Difficulties of Novice Programmers. 10Th annual SIGCSE conference on Innovation an technology in computer science education ItiCSE '05

Lovos, E. (2012). Revisión de Herramientas Colaborativas para la Enseñanza de la Programación a Alumnos Novatos. First Argentine Conference on Human - Computer Interaction, Telecommunications, Informatics and Scientific Information - HCITISI 201. Córdoba, Argentina, noviembre 2012

- (2014). El uso de estrategias colaborativas mediadas por tecnología. La enseñanza de la programación en el primer año de la Licenciatura en Sistemas de la UNRN (Tesis de Maestría). Universidad Nacional de La Plata. Disponible en: http://sedici.unlp.edu.ar/handle/10915/38038

Lucero, M. (2003). Entre el trabajo colaborativo y el aprendizaje colaborativo. Revista Iberoamericana de Educación, 36(1), pp. 23-200. http://www.Capmpus.oei.org/revista/de los lectores/528/Lucero.pdf

Madoz, M.C., Gorga, G., Russo, C. (2005). Análisis del Impacto de las TIC's en el proceso de aprendizaje de alumnos universitarios de nivel inicial. Congreso de Tecnologías de la Información y la Comunicación en la Enseñanza de las Ciencias. TICEC 05. La Plata, 29 de Septiembre del 2005.

Maldonado Pérez, M. (2007). El trabajo colaborativo en el aula universitaria. Revista Laurus, 13(23). Universidad Pedagógica Experimental Libertador. Caracas Venezuela.

Matthíasdóttir, Á.(2006). How to teach programming languages to novice students? Lecturing or not? Proceedings of the International Conference on Computer Systems and Technologies, June 15-16, University of Veliko Tarnovo, 2006, Bulgaria.

Moodle. https://moodle.org/

Pérez Pérez, J. R., Paule Ruiz, J.M., Del Puerto M., Cueva Lovelle J. M. (2006). Capitulo 3. Sistemas orientados a la mejorade la calidad del software. En congreso IV International Confe- 
rence on Multimedia and Information \& Communication Technologies in Education (mICTE2006)

Restrepo Gómez, B. (2005). Aprendizaje basado en problemas (ABP): una innovación didáctica para la enseñanza universitaria. Educadores, 8, pp. 9-19. Disponible en: redalyc.uaemex. $\mathrm{mx} /$ redalyc/pdf/834/83400803.pdf. Junio 2012

Rodriiguez del Pino, J.C., Royo Rubio E., Hernandez Figueroa. (2010). VPL: Laboratorio virtual de programación para Moodle. En Actas de las XVI Jornadas de Enseñanza Universitaria de Informática, Jenui 2010, pags. 429-435, Santiago de Compostela, Julio 2010.

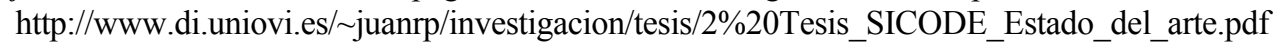

Teague, D. y Roe, P. (2008). Collaborative learning - towards a solution for novice programmers. In Proc. Tenth Australasian Computing Education Conference (ACE 2008), Wollongong, NSW, Australia. CRPIT, 78. Simon and Hamilton, M., Eds. ACS. 147-154.

Vygotsky. L. S. (1978). Mind in Society: The Development of Higher Psychological Processes. Cambirdge: Harvard University Press.

Zañartu Correa, L. M. (2003). Aprendizaje colaborativo: una nueva forma de Diálogo Interpersonal y en Red. Revista Digital de Educación y Nuevas Tecnologías, 5(28).

\section{SOBRE LA AUTORA}

Edith Lovos: Ingeniera de Sistemas de la Universidad Nacional del Centro de la Provincia de Buenos Aires. Obtuvo su Magister en Tecnología Informática Aplicada en Educación en la facultad de Informática de la Universidad Nacional de La Plata, en el año 2014. Se desempeña como docente del área de Algoritmos y Lenguajes en la carrera Lic. en Sistemas de la Universidad Nacional de Río Negro. Realiza investigación en relación a estrategias de enseñanza y aprendizaje que promuevan la colaboración y la auto-regulación de los aprendizajes en el nivel superior, haciendo uso de recursos tecnológicos. 